\title{
Wardowski Type Characterization of the Interpolative Berinde Weak Fixed Point Theorem
}

\author{
Clement Boateng Ampadu
}

31 Carrolton Road, Boston, MA 02132-6303, USA

e-mail: drampadu@hotmail.com

\begin{abstract}
In [1], Wardowski introduced the $F$-contractions, and used it to prove the Banach contraction principle. In this paper we introduce a concept of $F$-interpolative Berinde weak contraction, and use it to prove the interpolative Berinde weak mapping theorem of [2].
\end{abstract}

\section{Introduction and Preliminaries}

Definition 1.1. Let $F: \mathbb{R}_{+} \mapsto \mathbb{R}$ be a mapping satisfying

(a) $F$ is strictly increasing, that is, for all $\alpha, \beta \in \mathbb{R}_{+}$such that $\alpha<\beta, F(\alpha)<$ $F(\beta)$;

(b) For each sequence $\left\{\alpha_{n}\right\}_{n \in \mathbb{N}}$ of positive numbers, $\lim _{n \rightarrow \infty} \alpha_{n}=0$ iff $\lim _{n \rightarrow \infty} F\left(\alpha_{n}\right)=-\infty$;

(c) There exists $\lambda \in(0,1)$ such that $\lim _{\lambda \rightarrow 0^{+}} \alpha^{\lambda} F(\alpha)=0$.

A mapping $T: X \mapsto X$, will be called an $F$-interpolative Berinde weak contraction, if there exists $\tau>0$ such that for all $x, y, \in X, x, y \notin F i x(T)$

$$
d(x, y)>0 \Longrightarrow \tau+F(d(T x, T y)) \leq F\left(d(x, y)^{\frac{1}{2}} d(x, T x)^{\frac{1}{2}}\right) .
$$

Received: October 26, 2020; Accepted: November 8, 2020

2010 Mathematics Subject Classification: 47H10, 54H25.

Keywords and phrases: F-contraction, interpolative Berinde weak contraction, fixed point theorems. 
Example 1.2. Let $F: \mathbb{R}_{+} \mapsto \mathbb{R}$ be given by the formula $F(\alpha)=\ln \alpha$. Observe that $F$ satisfies (a), (b), and (c) of the previous definition for any $\lambda \in(0,1)$. Each mapping $T: X \mapsto X$ satisfying the implication in the previous definition is an $F$-interpolative Berinde weak contraction such that

$$
d(T x, T y) \leq e^{-\tau} d(x, y)^{\frac{1}{2}} d(x, T x)^{\frac{1}{2}}
$$

for all $x, y \in X, x, y \notin F i x(T), T x \neq T y$. Note that for all $x, y \in X, x, y \notin F i x(T)$ such that $T x=T y$, the inequality

$$
d(T x, T y) \leq e^{-\tau} d(x, y)^{\frac{1}{2}} d(x, T x)^{\frac{1}{2}}
$$

still holds, that is, $T$ is an interpolative Berinde weak contraction [2].

\section{Main Result}

Theorem 2.1. Let $(X, d)$ be a complete metric space, and let $T: X \mapsto X$ be a continuous F-interpolative Berinde weak contraction. Then $T$ has a fixed point $x^{*} \in X$, and for every $x_{0} \in X$, the sequence $\left\{T^{n} x_{0}\right\}_{n \in \mathbb{N}}$ is convergent to $x^{*}$.

Proof. Let $x_{0} \in X$ be arbitrary and fixed. Define a sequence $\left\{x_{n}\right\}_{n \in \mathbb{N}} \subset X$ by $x_{n+1}=T x_{n}, n=0,1,2, \cdots$. Denote $\gamma_{n}=d\left(x_{n}, x_{n+1}\right)$, for $n=0,1,2, \cdots$. If there exists $n_{0} \in \mathbb{N}$ for which $x_{n_{0}+1}=x_{n_{0}}$, then $x_{n_{0}}$ is a fixed point of $T$ and the proof is finished. So we assume $x_{n+1} \neq x_{n}$ for all $n \in \mathbb{N}$. Then $\gamma_{n}>0$ for all $n \in \mathbb{N}$. Since $T$ is an $F$-interpolative Berinde weak contraction, we deduce the following

$$
\begin{aligned}
\tau+F\left(\gamma_{n}\right) & =\tau+F\left(d\left(T x_{n-1}, T x_{n}\right)\right) \\
& \leq F\left(d\left(x_{n-1}, x_{n}\right)^{\frac{1}{2}} d\left(x_{n-1}, T x_{n-1}\right)^{\frac{1}{2}}\right) \\
& =F\left(d\left(x_{n-1}, x_{n}\right)^{\frac{1}{2}} d\left(x_{n-1}, x_{n}\right)^{\frac{1}{2}}\right) \\
& =F\left(d\left(x_{n-1}, x_{n}\right)\right) \\
& =F\left(\gamma_{n-1}\right) .
\end{aligned}
$$

which implies

$$
F\left(\gamma_{n}\right) \leq F\left(\gamma_{n-1}\right)-\tau \leq F\left(\gamma_{n-2}\right)-2 \tau \leq \cdots \leq F\left(\gamma_{0}\right)-n \tau .
$$


The above implies $\lim _{n \rightarrow \infty} F\left(\gamma_{n}\right)=-\infty$. It now follows from (b) of Definition 1.1, that $\lim _{n \rightarrow \infty} \gamma_{n}=0$. From (c) of Definition 1.1, there exists $\lambda \in(0,1)$ such that $\lim _{n \rightarrow \infty} \gamma_{n}^{\lambda} F\left(\gamma_{n}\right)=0$. Since

$$
F\left(\gamma_{n}\right) \leq F\left(\gamma_{n-1}\right)-\tau \leq F\left(\gamma_{n-2}\right)-2 \tau \leq \cdots \leq F\left(\gamma_{0}\right)-n \tau
$$

we deduce the following

$$
\begin{aligned}
\gamma_{n}^{\lambda} F\left(\gamma_{n}\right)-\gamma_{n}^{\lambda} F\left(\gamma_{0}\right) & \leq \gamma_{n}^{\lambda}\left(F\left(\gamma_{0}\right)-n \tau\right)-\gamma_{n}^{\lambda} F\left(\gamma_{0}\right) \\
& =-\gamma_{n}^{\lambda} n \tau \\
& \leq 0 .
\end{aligned}
$$

Since $\lim _{n \rightarrow \infty} \gamma_{n}=0$ and $\lim _{n \rightarrow \infty} \gamma_{n}^{\lambda} F\left(\gamma_{n}\right)=0$. If we take limits in the above inequality we deduce $\lim _{n \rightarrow \infty} n \gamma_{n}^{\lambda}=0$. This suggests there exists $n_{1} \in \mathbb{N}$ such that $n \gamma_{n}^{\lambda} \leq 1$ for all $n \geq n_{1}$. Consequently, we have

$$
\gamma_{n} \leq \frac{1}{n^{\frac{1}{\lambda}}}
$$

for all $n \geq 1$. Now we show that $\left\{x_{n}\right\}$ is Cauchy. Consider, $m, n \in \mathbb{N}$ such that $m>n \geq n_{1}$. From the definition of the metric and the above inequality, we get

$$
\begin{aligned}
d\left(x_{m}, x_{n}\right) & \leq \gamma_{m-1}+\gamma_{m-2}+\cdots+\gamma_{m} \\
& \leq \sum_{i=n}^{\infty} \gamma_{i} \\
& \leq \sum_{i=n}^{\infty} \frac{1}{i^{\frac{1}{\lambda}}} .
\end{aligned}
$$

Since $\sum_{i=1}^{\infty} \frac{1}{i^{\frac{1}{\lambda}}}$ is convergent, it follows from the above inequality that $\left\{x_{n}\right\}$ is a Cauchy sequence. From the completeness of $X$, there exists $x^{*} \in X$ such that $\lim _{n \rightarrow \infty} x_{n}=x^{*}$. Finally, since $T$ is continuous we deduce the following

$$
d\left(T x^{*}, x^{*}\right)=\lim _{n \rightarrow \infty} d\left(T x_{n}, x_{n}\right)=\lim _{n \rightarrow \infty} d\left(x_{n+1}, x_{n}\right)=0
$$

which implies $x^{*}$ is a fixed point of $T$, and the proof is finished. 


\section{References}

[1] Dariusz Wardowski, Fixed points of a new type of contractive mappings in complete metric spaces, Fixed Point Theory Appl. 2012 (2012), Article No. 94.

https://doi.org/10.1186/1687-1812-2012-94

[2] Clement Boateng Ampadu, Some fixed point theory results for the interpolative-Berinde weak operator, Earthline J. Math. Sci. 4(2) (2020), $253-271$.

https://doi.org/10.34198/ejms.4220.253271

This is an open access article distributed under the terms of the Creative Commons Attribution License (http://creativecommons.org/licenses/by/4.0/), which permits unrestricted, use, distribution and reproduction in any medium, or format for any purpose, even commercially provided the work is properly cited. 